\title{
Marine Alkaloids (-)-Pictamine and (-)-Lepadin B Block Neuronal Nicotinic Acetylcholine Receptors
}

\author{
Hiroshi Tsuneki, ${ }^{*, a}$ Yueren You ${ }^{a}$ Naoki ToyookA, ${ }^{b}$ Toshiyasu SASAokA,${ }^{a}$ Hideo Nemoto, ${ }^{b}$ \\ John A. DANI, ${ }^{c}$ and Ikuko KIMURA ${ }^{a}$ \\ ${ }^{a}$ Department of Clinical Pharmacology, Toyama Medical and Pharmaceutical University; ${ }^{b}$ Faculty of Pharmaceutical \\ Sciences, Toyama Medical and Pharmaceutical University; 2630 Sugitani, Toyama 930-0194, Japan: and ${ }^{c}$ Department of \\ Neuroscience, Baylor College of Medicine; Houston, TX, 77030-3498 U.S.A. \\ Received October 12, 2004; accepted January 18, 2005; published online January 21, 2005
}

Ascidians (sea squirts) contain a wealth of alkaloids, but their influence over neuronal nicotinic acetylcholine receptors (nAChRs) has not been evaluated. In this study, we examined the effects of two synthetic compounds, (-)-pictamine, a quinolizidine alkaloid from Clavelina picta, and (-)-lepadin B, a decahydroquinoline alkaloid from Clavelina lepadiformis, on major types of neuronal nicotinic receptors $(\alpha 4 \beta 2$ and $\alpha 7)$ expressed in Xenopus oocytes. We found that these alkaloids are potent blockers at these receptors: acetylcholine-elicited currents through $\alpha 4 \beta 2$ and $\alpha 7$ receptors were blocked by (-)-pictamine with $\mathrm{IC}_{50}$ values of $1.5 \mu_{\mathrm{M}}$ and $1.3 \mu_{\mathrm{M}}$, respectively, and by (-)-lepadin B with $\mathrm{IC}_{50}$ values of $0.9 \mu \mathrm{M}$ and $0.7 \mu \mathrm{M}$, respectively. Interestingly, no recovery was observed after the removal of (-)-pictamine in oocytes expressing $\alpha 4 \beta 2$ receptors, whereas the inhibited $\alpha 7$ currents quickly recovered after the removal of $(-)$-pictamine. Since there are few compounds that elicit irreversible blocks of $\alpha 4 \beta 2$ receptors, (-)-pictamine will be a novel, valuable tool to remove the $\alpha 4 \beta 2$-nAChR action from neuronal activities mediated by these two major types of nAChRs.

Key words nicotinic receptor; ascidians alkaloid; pictamine; Xenopus oocyte

Neuronal nicotinic acetylcholine receptors (nAChRs) are ligand-gated ion channels that mediate and/or modulate synaptic transmission in the nervous system. ${ }^{1-3)}$ In mammalian brain, neuronal nAChR subunits are identified as $\alpha 2-\alpha 7, \alpha 9, \alpha 10$, and $\beta 2-\beta 44^{4,5)}$ Ligand-binding studies indicate that the most abundant native nAChRs in the central nervous system are $\alpha 4 \beta 2$ heteromeric receptors and $\alpha 7$ homomeric receptors. $\left.{ }^{6}\right)$ Although nAChRs have been implicated in several neurological disorders including nicotinic addiction, epilepsy, Parkinson's disease and Alzheimer's disease, ${ }^{7-9)}$ nicotinic ligands and nicotinic-based therapies have only slowly and minimally become available. ${ }^{10-13)}$

A variety of alkaloids were isolated from amphibian skins, including indolizidines, quinolizidines, decahydroquinolines and piperidines, some of which have been identified as noncompetitive blockers of nAChRs at neuromuscular junction and in ganglionic PC12 cells ${ }^{14)}$ and recombinant neuronal nAChRs expressed in Xenopus oocytes. ${ }^{15)}$ To date, a wide range of toxins, including different types of decahydroquinolines and quinolizidines, have been isolated from ascidians (tunicates, subphylum Urochordata, class Ascidiacea) commonly known as sea squirts. ${ }^{16)}$ Nevertheless, none of the ascidian alkaloids has been tested in pharmacological research to develop therapeutically useful nicotinic drugs. Previously, we have redefined the absolute configuration of two ascidian alkaloids by enantioselective total synthesis: (-)-pictamine, a quinolizidine alkaloid from Clavelina picta, and (-)-lepadin B, a decahydroquinoline alkaloid from Clavelina lepadiformis. In the present study, we investigated the effects of $(-)$-pictamine and (-)-lepadin B on the major types of nicotinic receptors ( $\alpha 4 \beta 2$ and $\alpha 7)$ expressed in Xenopus oocytes.

\section{MATERIALS AND METHODS}

Materials All alkaloids used in this study were synthesized and are shown in Fig. 1. The synthesis of (-)-picta- mine and (-)-lepadin B was performed as reported previously. ${ }^{17,18)}$ The 3-piperidinol alkaloid (+)-prosafrinine, originally isolated from the leaves of Prosopis africana, has also been synthesized as described previously. ${ }^{19)}$ The structures of these alkaloids are identical to those of the natural products. The other reagents were purchased from Sigma (St. Louis, MO, U.S.A.), unless indicated otherwise.

Expression in Xenopus Oocytes To assess the nAChR activity, we measured current through the receptor-channel following the techniques described in detail by Tsuneki et al. ${ }^{15)}$ In brief, oocytes were isolated from Xenopus laevis frogs and were microinjected with $20 \mathrm{ng}$ cDNAs in the nucleus. The mixture of cDNAs of mouse $\alpha 4$ and mouse $\beta 2$ subunits was injected in a ratio of $1: 1$, whereas mouse $\alpha 7$ cDNA was injected alone. Oocytes were used in electrophysiological recordings 3 to $8 \mathrm{~d}$ after the injection. All experiments were carried out in accordance with guidelines approved by the Toyama Medical and Pharmaceutical Univer-

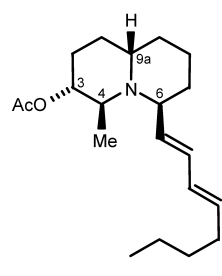

(-)-pictamine

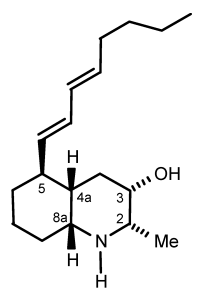

(-)-lepadin B

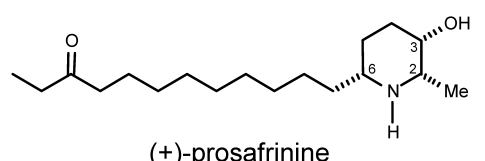

Fig. 1. Structures of (-)-Pictamine, (-)-Lepadin B and (+)-Prosafrinine Absolute stereochemistry is as follows, (-)-pictamine: $3 R, 4 S, 6 S, 9 \mathrm{a} S ;(-)$-lepadin B: $2 S, 3 S, 4 \mathrm{a} S, 5 S, 8 \mathrm{a} R ;(+)$-prosafrinine: $2 S, 3 S, 6 R$ 
sity Animal Research Committee.

Electrophysiological Recording An oocyte was placed in a $300-\mu 1$ tube-like chamber in which a low- $\mathrm{Ca}^{2+}$ Ringer's solution (82.5 mM NaCl, 2.5 mM KCl, $0.5 \mathrm{~mm} \mathrm{CaCl}_{2}, 2 \mathrm{~mm}$ $\mathrm{MgCl}_{2}$, and $5 \mathrm{~mm}$ HEPES, pH 7.4) containing $1 \mu \mathrm{M}$ atropine to block endogenous muscarinic receptors was perfused by gravity $(15 \mathrm{ml} / \mathrm{min})$. The low- $\mathrm{Ca}^{2+}$ Ringer's solution is used to prevent strong activation of the endogenous calcium-dependent chloride conductance. ${ }^{20}$ ) Current responses were recorded under two-electrode voltage-clamp at a holding potential of $-60 \mathrm{mV}$ using a GeneClamp 500 amplifier and pClamp7 software (Axon Instruments, Union City, CA, U.S.A.). The sampling rate was $20 \mathrm{~Hz}$. Electrodes contained $3 \mathrm{M} \mathrm{KCl}$ and had resistances of $<1 \mathrm{M} \Omega$. The rapid solution exchange was performed by a computer-controlled three-way Teflon solenoid valve (Parker Hannifin Corp., Division of General Valve, NJ, U.S.A.). Curve fittings to the concentration-inhibition curves for alkaloids (antagonists) and to the ACh concentration-response curves were performed as described previously. ${ }^{15)}$

\section{RESULTS}

Concentrations near the $30 \%$ effective doses $\left(\mathrm{EC}_{30}\right)$ of $\mathrm{ACh}$ were used to elicit the currents, that is, $1 \mu \mathrm{M}$ for $\alpha 4 \beta 2$ receptors and $100 \mu \mathrm{M}$ for $\alpha 7$ receptors. The $\alpha 4 \beta 2$ currents were inhibited by dihydro- $\beta$-erythroidine (the best characterized antagonist of $\alpha 4 \beta 2$ receptors, $5 \mu \mathrm{M}$ ) and $\alpha 7$ currents were inhibited by methyllycaconitine (a selective antagonist of $\alpha 7$ receptors, $10 \mathrm{~nm}$ ) as reported previously. ${ }^{15)}$ When the oocytes expressing $\alpha 4 \beta 2$ or $\alpha 7$ receptors were preincubated with (-)-pictamine $(3 \mu \mathrm{M})$ for $3 \mathrm{~min}$, the peak amplitude of currents elicited by ACh $(1 \mu \mathrm{M}$ for $\alpha 4 \beta 2$ receptors, $100 \mu \mathrm{M}$ for $\alpha 7$ receptors) was markedly decreased (Fig. 2A). Interestingly, no recovery was observed after the removal of (-)-pictamine in oocytes expressing $\alpha 4 \beta 2$ receptors, whereas the inhibited $\alpha 7$ currents completely recovered 12 min after the removal of (-)-pictamine. To quantitatively assess this profile, the oocytes expressing $\alpha 4 \beta 2$ receptors were stimulated with $\mathrm{ACh}$ pulses $(1 \mu \mathrm{M}, 5 \mathrm{~s})$ at indicated times (Fig. 2B). In the absence of the alkaloid, repeatable, constant currents were obtained. (-)-Pictamine $(3 \mu \mathrm{M})$ caused a timedependent blockade of the $\alpha 4 \beta 2$ currents, and the degree of blockade was maintained at least $48 \mathrm{~min}$ after the washout of (-)-pictamine (Fig. 2B). These results suggest that the rate of dissociation of $(-)$-pictamine is extremely different between $\alpha 4 \beta 2$ and $\alpha 7$ receptors. To compare the potency of (-)-pictamine on $\alpha 4 \beta 2$ and $\alpha 7$ currents, the peak amplitude of the currents was measured after $3 \mathrm{~min}$ of the alkaloid incubation. We observed that ( - -pictamine blocked the currents in a concentration-dependent manner, with $\mathrm{IC}_{50}$ values of $1.5 \mu \mathrm{M}$ for $\alpha 4 \beta 2$ currents and $1.3 \mu \mathrm{M}$ for $\alpha 7$ currents (Figs. $2 \mathrm{C}$, D, Table 1). Thus, (-)-pictamine equally blocks $\alpha 4 \beta 2$ and $\alpha 7-\mathrm{nAChRs}$. As shown in Fig. 2E, the concentration-response relationship for $\mathrm{ACh}$ on $\alpha 7$ receptors was fit by a single Hill equation $\left(\mathrm{EC}_{50}=158 \mu \mathrm{M}\right.$, Hill coefficient $\mathrm{nH}=1.31)$. The ACh concentration-response curve shifted downward in the presence of $(-)$-pictamine $(3 \mu \mathrm{M})$ (Fig. 2E), indicating that $(-)$-pictamine is not a competitive blocker of $\alpha 7$ receptors.

When the oocytes expressing $\alpha 4 \beta 2$ receptors were prein-
A
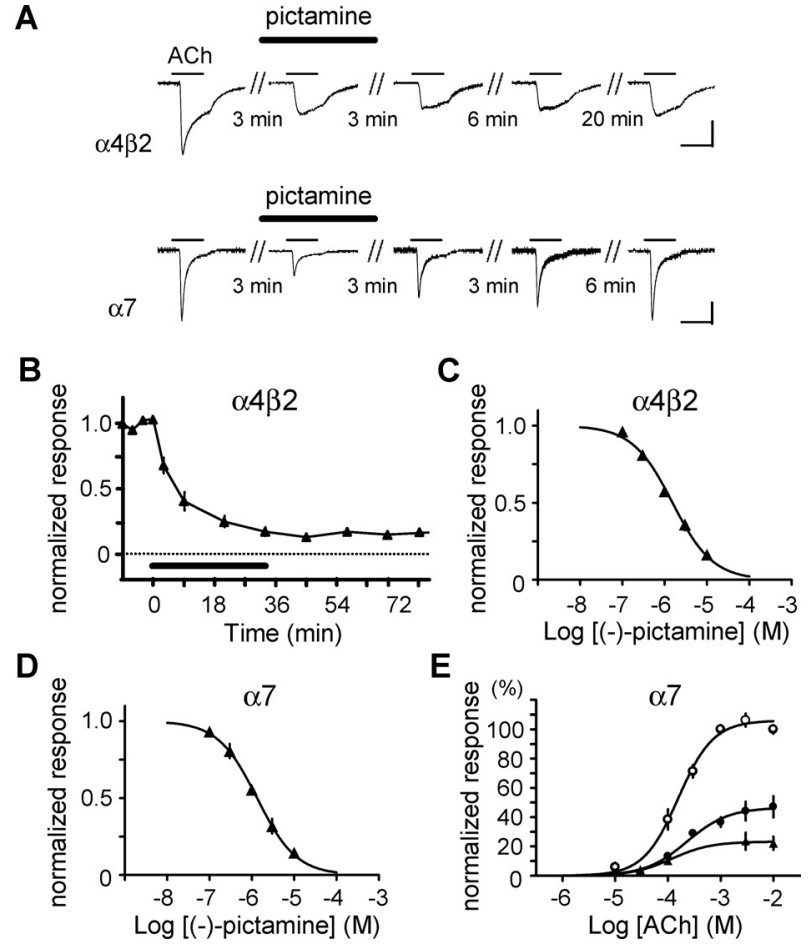

Fig. 2. Irreversible Inhibitory Effects of (-)-Pictamine on ACh-Induced Currents in Xenopus Oocytes Expressing $\alpha 4 \beta 2$ Nicotinic Receptors

Currents were recorded in voltage-clamp mode at $-60 \mathrm{mV}$. (A) Typical traces showing inhibition by (-)-pictamine $(3 \mu \mathrm{M})$ on $\alpha 4 \beta 2$ currents elicited by $1 \mu \mathrm{M} \mathrm{ACh}$ and $\alpha 7$ currents elicited by $100 \mu \mathrm{M}$ ACh. Horizontal bars indicate the period of perfusion with $\mathrm{ACh}$ for $5 \mathrm{~s}$. Vertical scale bars represent $300 \mathrm{nA}$ for $\alpha 4 \beta 2$ currents, and $150 \mathrm{nA}$ for $\alpha 7$

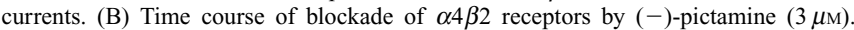
Each point represents the mean \pm S.E.M. of five consecutive ACh responses that were normalized to the initial current evoked by $1 \mu \mathrm{m}$ ACh alone recorded in the same oocyte. The horizontal bar indicates the time of pictamine superfusion. Concentration-response curves for (-)-pictamine on $\alpha 4 \beta 2$ (C) and $\alpha 7$ receptors (D). AChelicited currents were measured before and $3 \mathrm{~min}$ after the $(-)$-pictamine treatment. The current responses to $\mathrm{ACh}$ in the presence of $(-)$-pictamine in each oocyte were normalized to the ACh responses (control responses) recorded in the same oocytes. Values represent the mean \pm S.E.M. for five to six separate experiments. (E) Concentration-response curves for ACh on $\alpha 7 \mathrm{nAChRs}$ in the absence $(\bigcirc)$ and presence of alkaloids. Oocytes were preincubated with either $(-)$-pictamine $(3 \mu \mathrm{M}, \mathbf{\Delta})$ or $(-)$-lepadin B $(1 \mu \mathrm{M}, 0)$ for $3 \mathrm{~min}$, and then exposed to $\mathrm{ACh}(10 \mu \mathrm{M}$ to $10 \mathrm{~mm})$ in the presence of alkaloids. Each current response to $\mathrm{ACh}$ in the presence of the alkaloids was normalized to the maximal current evoked by $1 \mathrm{~mm} \mathrm{ACh}$ alone recorded in the same oocyte. Values represent the mean \pm S.E.M. for five to eight separate experiments.

Table 1. Potency of Blocking Effects of Alkaloids on Xenopus Oocytes Expressing Recombinant Nicotinic Receptors

\begin{tabular}{lccc}
\hline \hline & \multicolumn{2}{c}{$\mathrm{IC}_{50}(95 \%$ confidence intervals $), \mu \mathrm{M}$} & \\
\cline { 2 - 3 } & $\alpha 4 \beta 2$ & $n$ \\
& $1 \mu \mathrm{M} \mathrm{ACh}$ & $100 \mu \mathrm{M} \mathrm{ACh}$ & \\
\hline (-)-Pictamine & 1.5 & 1.3 & $5-6$ \\
& $(1.3,1.8)$ & $(1.1,1.6)$ & \\
$(-)$-Lepadin B & 0.9 & 0.7 & $5-8$ \\
& $(0.7,1.2)$ & $(0.5,0.9)$ & \\
(+)-Prosafrinine & 2.9 & 1.1 & 3 \\
& $(2.2,3.8)$ & $(0.7,1.9)$ &
\end{tabular}

cubated with (-)-lepadin B ( $3 \mu \mathrm{M})$ for 3 min, the peak amplitude of currents elicited by ACh $(1 \mu \mathrm{M})$ was greatly decreased (Fig. 3A). This blocking effect was reversible within 9 min. Similar reversible blockade was observed in ACh $(100 \mu \mathrm{M})$-elicited currents in oocytes expressing $\alpha 7$ receptors. In fact, these currents were blocked by this alkaloid in a 


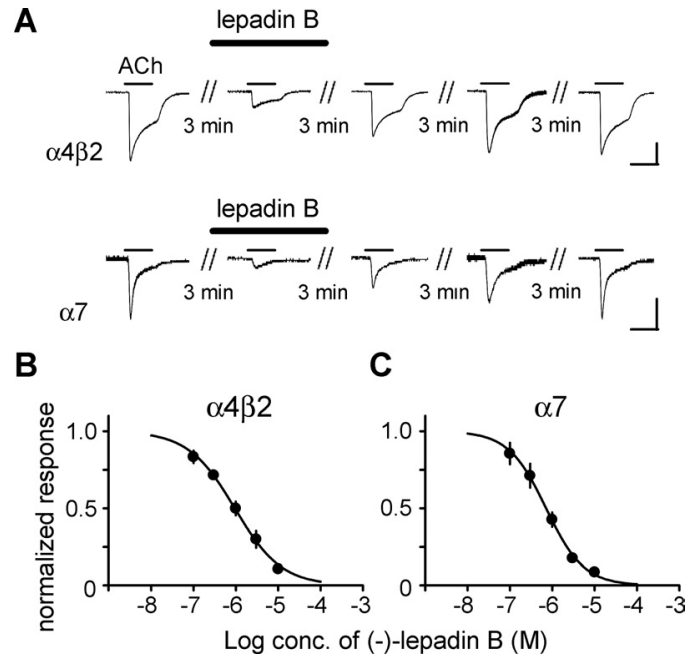

Fig. 3. Inhibitory Effects of (-)-Lepadin B on ACh-Induced Currents in Xenopus Oocytes Expressing Recombinant Nicotinic Receptors

Currents were recorded in voltage-clamp mode at $-60 \mathrm{mV}$. (A) typical traces showing inhibition by (-)-lepadin B $(3 \mu \mathrm{M})$ of $\alpha 4 \beta 2$ currents elicited by $1 \mu \mathrm{M}$ ACh and $\alpha 7$ currents elicited by $100 \mu \mathrm{M}$ ACh. Horizontal bars indicate the period of perfusion with $\mathrm{ACh}$ for $5 \mathrm{~s}$. Vertical scale bars represent $300 \mathrm{nA}$. The ACh-elicited currents recovered after removal of lepadin B. Concentration-response curves for (-)-lepadin B on $\alpha 4 \beta 2$ (B) and $\alpha 7$ receptors $(\mathrm{C})$. ACh-elicited currents were measured before and $3 \mathrm{~min}$ after the (-)-lepadin B treatment. The current responses to ACh in the presence of (-)-lepadin $\mathrm{B}$ in each oocyte were normalized to the $\mathrm{ACh}$ responses (control responses) recorded in the same oocytes. Values represent the mean \pm S.E.M. for five to eight separate experiments.

concentration-dependent manner, with $\mathrm{IC}_{50}$ values of $0.9 \mu \mathrm{M}$ for $\alpha 4 \beta 2$ currents and $0.7 \mu \mathrm{M}$ for $\alpha 7$ currents (Figs. 3B, C, Table 1). Thus, (-)-lepadin B appears to equally block $\alpha 4 \beta 2$ - and $\alpha 7$-nAChRs. Furthermore, as shown in Fig. 2E, the concentration-response curve for ACh on $\alpha 7$ receptors shifted downward in the presence of (-)-lepadin B $(1 \mu \mathrm{M})$, and the responses to $\mathrm{ACh}$ at the maximal concentrations (3 and $10 \mathrm{~mm}$ ) did not reach the levels observed in the absence of (-)-lepadin B. These results indicate that (-)-lepadin B is not a competitive blocker of $\alpha 7$ receptors.

We further examined the effect of (+)-prosafrinine on the ACh-elicited currents because the 3-hydroxy piperidine moiety is a structure that $(-)$-lepadin $\mathrm{B}$ and $(+)$-prosafrinine have in common. When the oocytes expressing $\alpha 4 \beta 2$ or $\alpha 7$ receptors were preincubated with (+)-prosafrinine for $3 \mathrm{~min}$, the peak amplitude of currents elicited by ACh $(1 \mu \mathrm{M}$ for $\alpha 4 \beta 2$ receptors, $100 \mu \mathrm{M}$ for $\alpha 7$ receptors) was markedly blocked: the $\mathrm{IC}_{50}$ values for $\alpha 4 \beta 2$ and $\alpha 7$ receptors were $2.9 \mu \mathrm{m}$ and $1.1 \mu \mathrm{M}$, respectively (Table 1).

\section{DISCUSSION}

In the present study, we found that ascidian alkaloids (-)pictamine and (-)-lepadin B are potent blockers of $\alpha 4 \beta 2$ and $\alpha 7$-nAChRs. Moreover, it is interesting that no recovery of $\alpha 4 \beta 2$ currents was observed after the removal of (-)-pictamine, whereas the inhibited $\alpha 7$ currents quickly recovered after its removal. It is possible that "treatment and washout" of (-)-pictamine would effectively remove the $\alpha 4 \beta 2$-nAChR action from neuronal activities mediated mainly by $\alpha 4 \beta 2$ and $\alpha 7$ receptors, such as dopaminergic neuron activities in mouse ventral tegmental area and substantia nigra pars compacta. ${ }^{21)}$ Further studies are, however, required to determine the selectivity of (-)-pictamine for $\alpha 4 \beta 2$ receptors over several other subtypes of nAChRs, including muscle-type nAChRs. Thus, this study is the first report indicating that natural products derived from ascidians may be an overlooked source for the discovery of novel nicotinic ligands.

The present results demonstrate that $(-)$-pictamine is a noncompetitive blocker of $\alpha 7$-nAChR, although the mechanisms underlying the long-term inhibition of $\alpha 4 \beta 2$-nAChR remains unclear. Typical noncompetitive blockers are channel blockers, whereas a few drugs, such as steroids, act noncompetitively (allosterically) at extracellular sites to inhibit nAChR function. ${ }^{9)}$ Several high affinity binding sites for the channel blockers have been found to be located in the walls of the ion channel, which are made up of transmembrane segments M2. ${ }^{22)}$ The amino acid sequences in and around M2 segments are very similar, but still not identical among $\alpha 4, \beta 2$ and $\alpha 7$ nicotinic subunits. ${ }^{22,23)}$ Since the lining of the ion channels is considered to be one of the most plausible binding sites of (-)-pictamine, we suggest that the diversity in the amino acid sequences may affect the stability of binding to each receptor subtype. We could not, however, exclude alternative possibility that (-)-pictamine irreversibly binds to the ACh-binding sites or their vicinity on $\alpha 4 \beta 2$-nAChR, and blocks the receptor activation. The snake toxin $\alpha$-bungarotoxin is a specific and almost irreversible antagonist of $\alpha 7$-nAChR. ${ }^{24)}$ Based on these properties, $\alpha$-bungarotoxin covalently linked to a resin is used for affinity chromatography to purify the $\alpha 7$ receptor protein. ${ }^{25)}$ Since there are few irreversible antagonists of $\alpha 4 \beta 2$ receptors, (-)-pictamine will be a novel, valuable tool to study the properties and functions of $\alpha 4 \beta 2$-nAChR.

Lepadin B has a unique structure, 2,5-disubstituted-3-hydroxy-decahydroquinoline. A number of 2,5-disubstituted decahydroquinolines have been isolated from skin extracts from dendrobatid frogs, ${ }^{14)}$ but no alkaloids from the frog skins have been classified as 2,5-disubstituted-3-hydroxydecahydroquinolines. Since there is a 3-hydroxy piperidine moiety in the structure of lepadin B, we further examined the effect of a 2,6-disubstituted-3-hydroxy-piperidine analog, (+)-prosafrinine, and found that it blocks both $\alpha 4 \beta 2$ - and $\alpha 7$-nAChRs. From the frog alkaloids, no such analog has been found (but 2,6-disubstituted-4-hydroxy-piperidine 241D that is a potent noncompetitive blocker of neuromuscular and ganglionic-types of nAChRs), ${ }^{14)}$ and, to our knowledge, this is the first report demonstrating the blocking effect of the 3hydroxylated piperidine on nAChRs. Although more detailed studies are required using other compounds of similar structure, the result suggests that the 3-hydroxy piperidine moiety of lepadin B may be an important structure for the interact with these nAChRs types. In addition, we consider that the 2,5-disubstituted decahydroquinoline moiety may also be a key structure for the biological activity of lepadin B, because several 2,5-disubstituted decahydroquinoline alkaloids, such as cis-195A, are reported to cause noncompetitive blockade of muscle-type nAChRs at the neuromuscular junction and ganglionic-type nAChRs in PC12 cells. ${ }^{14)}$ Similarly, lepadin $\mathrm{B}$ is predicted to be a noncompetitive blocker of several subtypes of nAChRs, including muscle-type nAChRs, and these pharmacological profiles needs to be elucidated in a future study.

Naturally occurring toxins are thought to be used by the 
sea squirts for chemical defense. ${ }^{26)}$ It has been reported that lepadin B elicits cytotoxic effects with $\mathrm{IC}_{50}$ values of $44 \mu \mathrm{M}$ for human brest cancer MCF7, $26 \mu \mathrm{M}$ for glioblastoma U373, and $38 \mu \mathrm{M}$ for ovarian carcinoma HEY cells. ${ }^{27)}$ However, these toxic concentrations are 30- to 60 -fold higher than the $\mathrm{IC}_{50}$ values of (-)-lepadin B for blockade of the nAChRs expressed in oocytes (Table 1). In fact, the blocking effect of (-)-lepadin B was reversible and reproducible in the same oocytes (data not shown). Moreover, no changes in the holding current were observed during the application of (-)-lepadin B (data not shown). Therefore, it is not likely that (-)lepadin B damages oocytes in the present condition.

Recent genetic studies demonstrate that autosomal-dominant nocturnal frontal lobe epilepsy (ADNFLE) is associated with a mutation in the $\alpha 4$ or the $\beta 2$ subunit of nAChRs. ${ }^{9}$ The mutant $\mathrm{nAChR}$ expressed in Xenopus oocytes exhibits an increased sensitivity to ACh. A promising therapeutic strategy suggested for ADNFLE is to reduce the enhanced responses of the mutant $\alpha 4 \beta 2-\mathrm{nAChRs}$ by noncompetitive nicotinic antagonists (open channel blockers). That type of blocker could selectively target the receptors displaying a gain of function by blocking them in a use-dependent manner. ${ }^{9)}$ Although (-)-pictamine does not appear to be an usedependent blocker, its longer-term inhibition suggests that further studies of ascidian alkaloids could reveal beneficial developments for nicotinic-based treatment of neurological diseases.

Acknowledgments The present work was supported in part by Smoking Research Foundation (Japan) (to I.K.) and by Grant-in-Aid (16590435) for Scientific Research from the Ministry of Education, Culture, Sports, Science and Technology, Japan (to H.T.). J.A.D. was supported by grants from the NINDS and NIDA of the National Institutes of Health (U.S.A.). We are grateful to Dr. Jerry A. Stitzel (University of Colorado) for providing us with plasmid DNA. We would also like to thank Dr. Hideki Sakai (Toyama Medical and Pharmaceutical University) for the continuous support on data acquisition.

\section{REFERENCES}

1) Changeux J. P., Bertrand D., Corringer P. J., Dehaene S., Edelstein S.,
Léna C., Le Novère N., Marubio L., Picciotto M., Zoli M., Brain Res. Brain Res. Rev., 26, 198-216 (1998).

2) Jones S., Sudweeks S., Yakel J. L., Trends Neurosci., 22, 555-561 (1999).

3) Dajas-Bailador F., Wonnacott S., Trends Pharmacol. Sci., 25, 317324 (2004).

4) Lindstrom J., "Ion Channels," Vol. 4, ed. by Narahashi T., Plenum Press, New York, 1996, pp. 377-450.

5) Elgoyhen A. B., Vetter D. E., Katz E., Rothlin C. V., Heinemann S. F., Boulter J., Proc. Natl. Acad. Sci. U.S.A., 98, 3501-3506 (2001).

6) Clarke P. B., Prog. Brain Res., 145, 253-260 (2004).

7) Weiland S., Bertrand D., Leonard S., Behav. Brain Res., 113, 43-56 (2000).

8) Dani, J. A., Biol. Psychiatry, 49, 166-174 (2001).

9) Hogg R. C., Bertrand D., Bioorg. Med. Chem. Lett., 14, 1859-1861 (2004).

10) Blesa R., Dement. Geriatr. Cogn. Disord., 11, (Suppl. 1), 28-34 (2000).

11) Maelicke A., Schrattenholz A., Samochocki M., Radina M., Albuquerque E. X., Behav. Brain Res., 113, 199-206 (2000).

12) Palmer A. M., Trends Pharmacol. Sci., 23, 426- 433 (2002).

13) Zhang L., Zhou F.-M., Dani J. A., Mol. Pharmacol., 66, 538-544 (2004).

14) Daly J. W., Garraffo H. M., Spande T. F., "Alkaloids: Chemical and Biological Perspectives," Vol. 13, ed. by Pelletier S. W., Pergamon Press, New York, 1999, pp. 1-161.

15) Tsuneki H., You Y., Toyooka N., Kagawa S., Kobayashi S., Sasaoka T., Nemoto H., Kimura I., Dani J. A., Mol. Pharmacol., 66, 1061-1069 (2004).

16) Faulkner D. J., Nat. Prod. Rep., 18, 1-49 (2001).

17) Toyooka N., Okumura M., Takahata H., Nemoto H., Tetrahedron, 55, 10673-10684 (1999).

18) Toyooka N., Yotsui Y., Yoshida Y., Momose T., Nemoto H., Tetrahedron, 55, 15209-15224 (1999).

19) Toyooka N., Yoshida Y., Yotsui Y., Momose T., J. Org. Chem., 64, 4914- 4919 (1999).

20) Vernino S., Amador M., Luetje C. W., Patrick J., Dani J. A., Neuron, 8, 127-134 (1992).

21) Champtiaux N., Gotti C., Cordero-Erausquin M., David D. J., Przybylski C., Léna C., Clementi F., Moretti M., Rossi F. M., Le Novère N., McIntosh J. M., Gardier A. M., Changeux J. P., J. Neurosci., 23, 7820-7829 (2003).

22) Galzi J. L., Changeux J. P., Neuropharmacology, 34, 563-582 (1995).

23) Rodrigues-Pinguet N., Jia L., Li M., Figl A., Klaassen A., Truong A., Lester H. A., Cohen B. N., J. Physiol., 550, 11-26 (2003).

24) Alkondon M., Albuquerque E. X., J. Pharmacol. Exp. Ther, 265, 1455-1473 (1993).

25) Ringler P., Kessler P., Menez A., Brisson A., Biochim. Biophys. Acta, 1324, 37-46 (1997).

26) Watters D. J., Van Den Brenk A. L., Toxicon, 31, 1349-1372 (1993).

27) Kubanek J., Williams D. E., Dilip de Silva E., Allen T., Andersen R. J., Tetrahedron Lett., 36, 6189—6192 (1995). 